\title{
Integration of Metal Organic Chemical Vapour Deposition and Wet Chemical Techniques to Obtain Highly Ordered Porous ZnO Nanoplatforms
}

\author{
Maria Elena Fragalà ${ }^{1, *}$, Yana Aleeva ${ }^{2}$, and Cristina Satriano ${ }^{1}$ \\ ${ }^{1}$ Dipartimento di Scienze Chimiche ed INSTM Udr Catania, Università di Catania, Viale Andrea Doria, 6-95125, Catania, Italy \\ ${ }^{2}$ Scuola Superiore di Catania, Dipartimento di Scienze Chimiche, Università di Catania, Viale Andrea Doria, 6-95125, Catania, Italy
}

\begin{abstract}
Large-area, highly ordered $\mathrm{ZnO}$ micropores-arrays consisting of $\mathrm{ZnO}$ nanotubes delimited by $\mathrm{ZnO}$ nanorods have been successfully fabricated and tested for protein sensing applications. ZnO seed layers have been deposited by Metal Organic Chemical Vapour Deposition and readily patterned by Colloidal Lithography to attain $\mathrm{ZnO}$ nanorods growth at selective sites by Chemical Bath Deposition. The used synthetic approach has been proven effective for the easy assembly of $\mathrm{ZnO}$ nanoplatforms into high-density arrays. Both patterned and unpatterned $\mathrm{ZnO}$ nanorods have been morphologically and compositionally characterised and, thus, tested for model studies of protein mobility at the interface. The patterned layers, having a higher contribution of surface polar moieties than the corresponding unpatterned surfaces, exhibit a reduced lateral diffusion of the adsorbed protein. This evidence is related to the intrinsic porous nature of the $\mathrm{ZnO}$ hemispherical arrays characterised by a nanotube-nanorod hybrid networks. The present study gives a great impetus to the fabrication of tunable $\mathrm{ZnO}$ nanoplatforms having multiple morphologies and exceptionally high surface areas suitable for application in sensing devices.
\end{abstract}

Keywords: ZnO, Nanoplatforms, Colloidal Lithography, Biosensing, FRAP.

\section{INTRODUCTION}

Nanomaterials have recently experienced great attention from the scientific communities for their multipotential applications. ${ }^{1}$ In particular, $\mathrm{ZnO}$ is actually considered a promising multifunctional material for a wide ensemble of applications ranging from surface acoustic wave devices, ${ }^{2}$ sensors, ${ }^{3}$ light-emitting diodes, ${ }^{4}$ solar cells ${ }^{5}$ and many other. Recently, one-dimensional (1D) $\mathrm{ZnO}$ nanomaterials have been extensively studied because both their functional properties and highly controllable morphology make them important building blocks for understanding nanoscale phenomena and realizing nanoscale devices. ${ }^{6}$ What is more, among the several advantages $\mathrm{ZnO}$-based materials, even when scaled down at nanometric dimensions, are to mention the non toxicity, the biosafety and the biocompatibility, that make $\mathrm{ZnO}$ nanostructured material an appealing nanoplatform for many medical and biological applications. ${ }^{7}$

In this paper, we report a new method to obtain $\mathrm{ZnO}$ nanoplatform on glass substrates by integration of Metal

\footnotetext{
*Author to whom correspondence should be addressed.
}

Organic Chemical Vapor Deposition (MOCVD) with Colloidal Lithography (CL) and Chemical Bath Deposition (CBD) approaches. ${ }^{8,9}$ It is worth mentioning that wet chemical approaches are currently attracting considerable efforts of research activity as bottom-up strategies to form one-dimensional $\mathrm{ZnO}$ nanostructures. In fact, wet chemistry based methods are versatile, low cost, environmental friendly techniques and allow large-scale production on almost every kind of substrates. ${ }^{10}$ In addition, the lowtemperature aqueous solution strategies used to grow $\mathrm{ZnO}$ nanostructures are fully compatible with emerging strategies devoted to the patterned growth of ordered $\mathrm{ZnO}$ nanostructure arrays by employing monolayer colloidal crystals. In fact, colloidal lithography has been proven to be a flexible and cost-effective technique for the patterning of nanostructured arrays with long-range periodicity in a large scale. ${ }^{11}$

$\mathrm{ZnO}$ nanoplatforms have been recently investigated to study protein adsorption processes and have demonstrated interesting potentialities as fluorescence based sensor. ${ }^{7,12}$

Herein, high surface area $\mathrm{ZnO}$ nanoplatforms were characterized by scanning electron microscopy (SEM) and X-ray photoelectron spectroscopy (XPS). The interaction 
of the newly deposited materials with a model biomolecule (fluorescein-labeled albumin) was investigated by fluorescence recovery after photobleaching (FRAP) experiments.

\section{EXPERIMENTAL DETAILS}

A reduced pressure horizontal hot wall MOCVD reactor was used to deposit $\mathrm{ZnO}$ seed layers on silicon and glass substrates. ${ }^{13} \mathrm{Ar}(250 \mathrm{sccm})$ and $\mathrm{O}_{2}(250 \mathrm{sccm})$ were used as carrier and reaction gases, respectively and the total pressure in the reactor was about 5 Torr. Depositions were carried out for 1 hour min and the deposition temperature is $600{ }^{\circ} \mathrm{C}$. After that, polystyrene (PS, Aldrich) microbeads of 1 micron of diameter were deposited onto the seed layers by drop casting and allowed to self-assemble in ordered close-packed colloidal monolayers. ${ }^{14}$ The optimal conditions to get the best monolayer coverage on the seed layer were found by changing the colloidal dispersion concentration (from 1 to $3 \% \mathrm{wt}$.) and the solvent mixture ratio (water/ethanol). The precursor solutions used for CBD growth were prepared by mixing $\mathrm{Zn}\left(\mathrm{CH}_{3} \mathrm{COO}\right)_{2} \cdot 2 \mathrm{H}_{2} \mathrm{O}$ with $\mathrm{N}, \mathrm{N}, \mathrm{N}^{\prime}, \mathrm{N}^{\prime}$-Tetramethylethylenediamine (TMEDA) water solutions while keeping a 1:1 constant volume ratio. ${ }^{15,16}$ All chemicals were analytical reagent grade and used without further purification. For all experiments focused on effect of concentration, the volume ratio of the two solutions has been kept constant at 1:1, while changing the concentrations to meet the experimental requirements. The CBD growth has been carried out at $70{ }^{\circ} \mathrm{C}$ in a glass beaker placed in an oil bath by immersing the pretreated substrates in the precursor solutions. The polymeric mask was removed by immersing the samples in toluene overnight.

Morphologies of $\mathrm{ZnO}$ nanostructures and nanoplatforms were investigated using a LEO Supra 55VP field emission scanning electron microscope (FEG-SEM). The X-ray photoelectron experiments (XPS) were carried out with a base pressure of $2 \times 10^{-10}$ Torr using a PHI ESCA/SAM 5600 Multytechnique spectrometer. A monochromatic $\mathrm{AlK} \alpha$ radiation source $(h \nu=1486.6 \mathrm{eV})$ was used. The surface analysis was made in both survey and narrow region scan modes, an incremental step size of $1 \mathrm{eV}$ for survey scans and $0.05 \mathrm{eV}$ for the narrow scans, and a $0.8 \mathrm{~mm}$ slit width, respectively. The high resolution photoelectron peaks of $\mathrm{Zn} 2 \mathrm{p}, \mathrm{C} 1 \mathrm{~s}, \mathrm{Si} 2 \mathrm{p}$ and $\mathrm{O} 1 \mathrm{~s}$ were recorded with $150 \mathrm{eV}$ pass energy. The XPS signals were analysed with a peak synthesis program assuming a nonlinear background and experimental bounds fitting by a combination of Gaussian (80\%) and Lorentzian (20\%) components. The atomic elemental compositions were evaluated using sensitivity factors provided by the F V5.4A software.

Fluorescein-isothiocyanate labelled albumin (FITC-alb, Sigma), $100 \mu \mathrm{g} / \mathrm{mL}$ in phosphate buffer saline solution (Sigma), was used as model protein to test the biomolecule

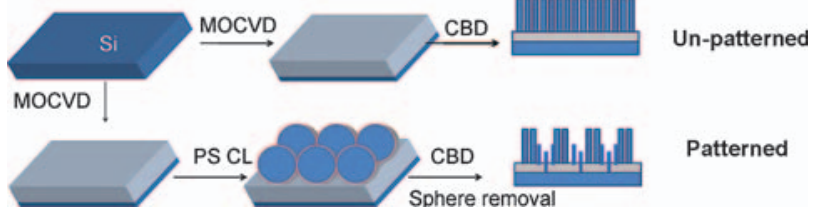

Scheme 1. Graphical representation of the experimental steps for the MOCVD-CL-CBD synthetical route for $\mathrm{ZnO}$ fabrication: (a) unpatterned $\mathrm{ZnO}$, (b) patterned $\mathrm{ZnO}$.

interaction with the $\mathrm{ZnO}$ nanoplatforms. FRAP experiments were performed on a laser scanning confocal microscope (Olympus FV1000 Fluoview) equipped with an oil immersion objective $(60 \times)$. The excitation was carried out with a multiline $\operatorname{Ar}$ laser $\left(\lambda_{\mathrm{ex}}=488 \mathrm{~nm}\right)$ and emitted light was detected at $572 \mathrm{~nm}$. FITC-alb was allowed to adsorb for $30 \mathrm{~min}$ on the substrates, then buffer was flowed to remove weakly bound proteins. At this point, a pattern with stripes of patterned and non patterned $\mathrm{ZnO}$ samples was bleached. The emission intensity values, measured for both unpatterned and patterned $\mathrm{ZnO}$ substrates after the protein adsorption, were measured before bleach,

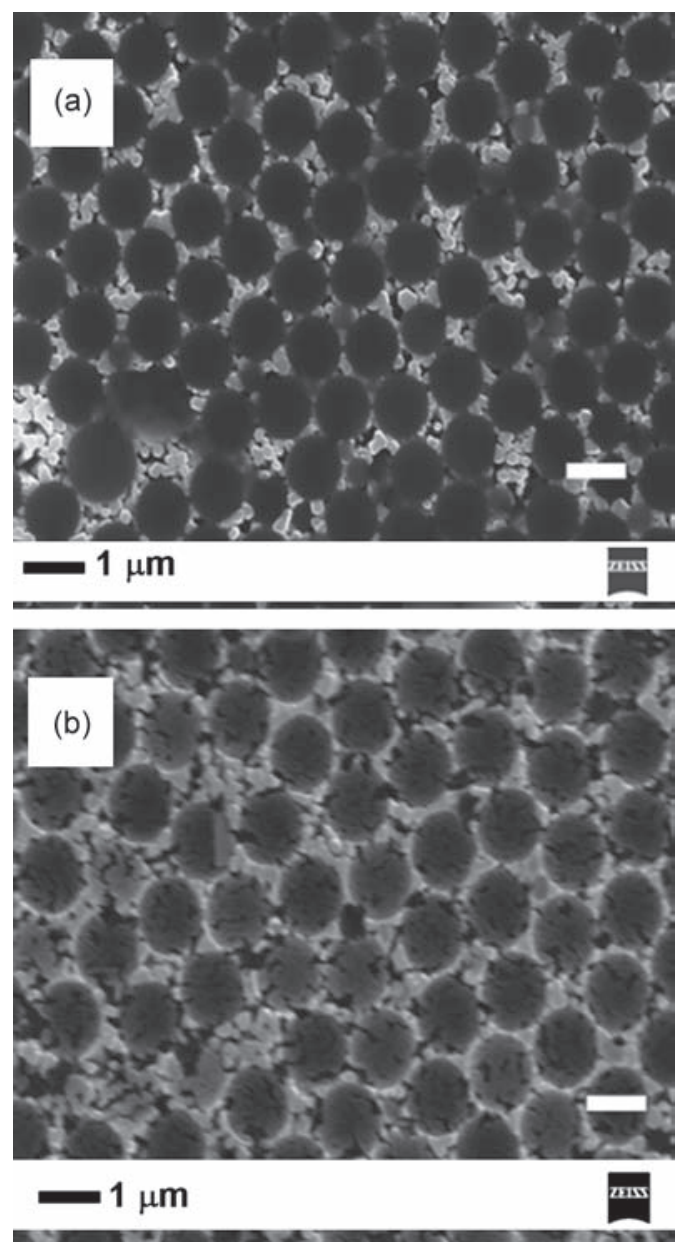

Fig. 1. SEM images of patterned $\mathrm{ZnO}$ nanorods before (a) and after (b) the PS microsphere removal. 
immediately after and after bleach every $5 \mathrm{~s}$ over a total acquisition interval of $90 \mathrm{~s}$.

\section{RESULTS AND DISCUSSION}

The fabrication of the present $\mathrm{ZnO}$ nanoplatforms requires the initial formation of $\mathrm{ZnO}$ seed, obtained by MOCVD, followed by self-assembly of PS microsphere masks and subsequent $\mathrm{CBD}$ growth of $\mathrm{ZnO}$ nanorods (Scheme 1).

Figure 1 shows the effective fabrication of patterned $\mathrm{ZnO}$ nanostructures both before (Fig. 1(a)) and after (Fig. 1(b)) the colloidal polymeric masks removal. It is well visible that the extension of the pattern vacancies within the template defines different density areas of $\mathrm{ZnO}$ nanorods, whose dimensions are strictly connected to the available surface area of the underlying seed layer. Despite the average variance of distance between PS spheres, wide
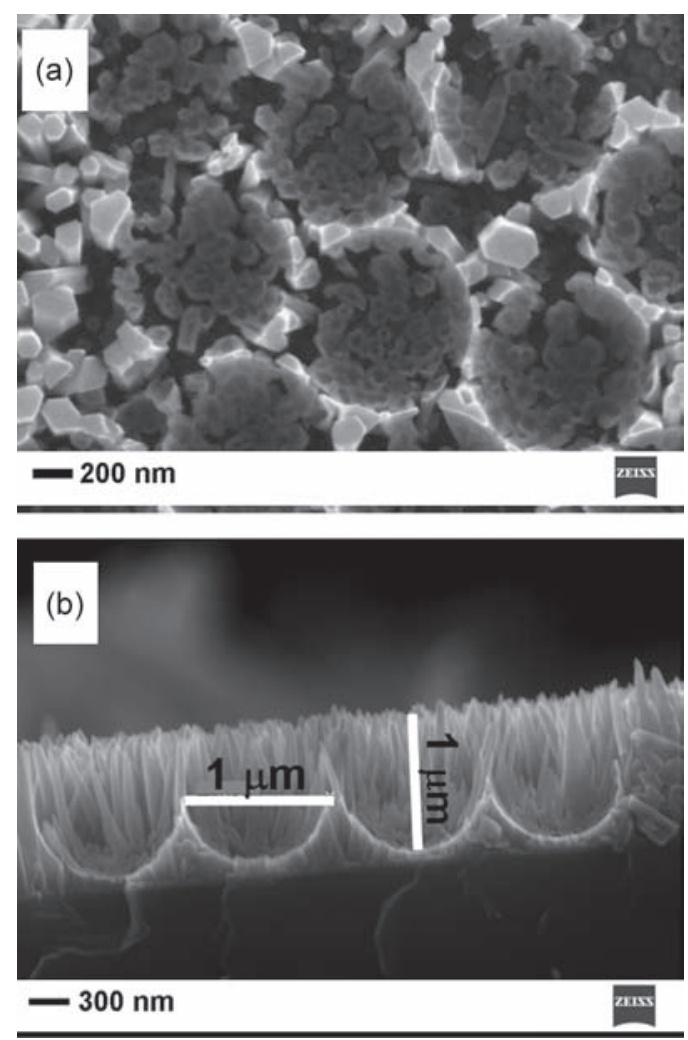

Fig. 2. SEM plan view of $\mathrm{ZnO}$ nanoplatforms inside the pores (a) and related cross section (b).

Table I. XPS quantitative analysis of patterned and unpatterned $\mathrm{ZnO}$ surfaces.

\begin{tabular}{lrrrr}
\hline & Zn & C & O & Si \\
\hline MOCVD & 23 & 35 & 43 & - \\
Unpatterned & 23 & 40 & 37 & - \\
Patterned & 7 & 38 & 43 & 12 \\
\hline
\end{tabular}

regions of densely packed hemispherical $\mathrm{ZnO}$ areas, confined by $\mathrm{ZnO}$ nanorods are available onto the patterned substrate. An insight at higher magnification inside the $\mathrm{ZnO}$ micropores (Fig. 2(a)) reveals the presence of $\mathrm{ZnO}$ nanotubes (mean diameter $\sim 40 \pm 20 \mathrm{~nm}$ ), as the result of a hindered growth process due to the confining effect of PS mask during the CBD growth. ${ }^{17}$ On the contrary, the external $\mathrm{ZnO}$ nanorods (mean diameter $\sim 100 \pm 30 \mathrm{~nm}$ ) are characterised by a higher density, better defined geometry and variable length according to the CBD growth parameters. ${ }^{15,16}$ From the SEM cross section (Fig. 2(b)) an average value of $\sim 600 \mathrm{~nm}$ for the $\mathrm{ZnO}$ pores depth is measured.

XPS analysis has been performed to study the surface composition of the obtained $\mathrm{ZnO}$ nanoplatforms (Table I). The comparison between patterned and unpatterned $\mathrm{ZnO}$ samples evidences significant differences of surface chemical composition, within the sampling depth at the current analysis conditions. In particular a dramatic decrease of detected zinc signal is found for patterned samples $(\sim 7 \%$ of atomic concentration vs. the $\sim 23 \%$ detected for both seed and unpatterned layers), associated to the appearance of the silicon signal from the substrate. This finding is likely related to the porous nature of the $\mathrm{ZnO}$ nanotubes confined inside the interior area of the micropore array (see Fig. 2(a)). In addition, solvent driven effects related
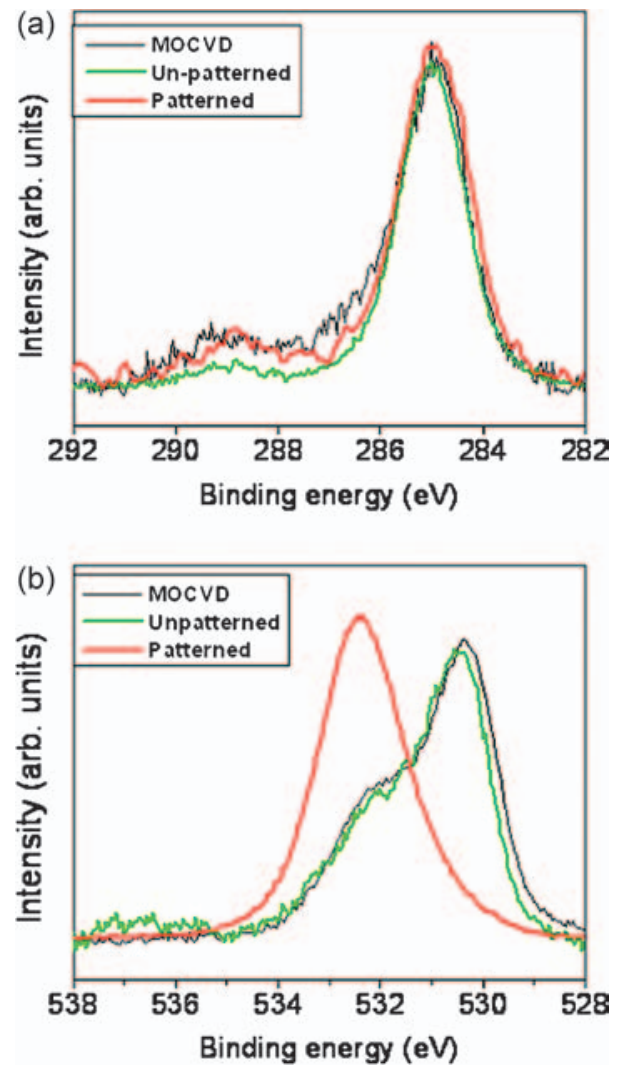

Fig. 3. XPS peaks of $\mathrm{C} 1 \mathrm{~s}$ (a) and $\mathrm{O} 1 \mathrm{~s}$ (b) for seed layer (MOCVD, black line), $\mathrm{ZnO}$ unpatterned (green line) and $\mathrm{ZnO}$ patterned (red line). 

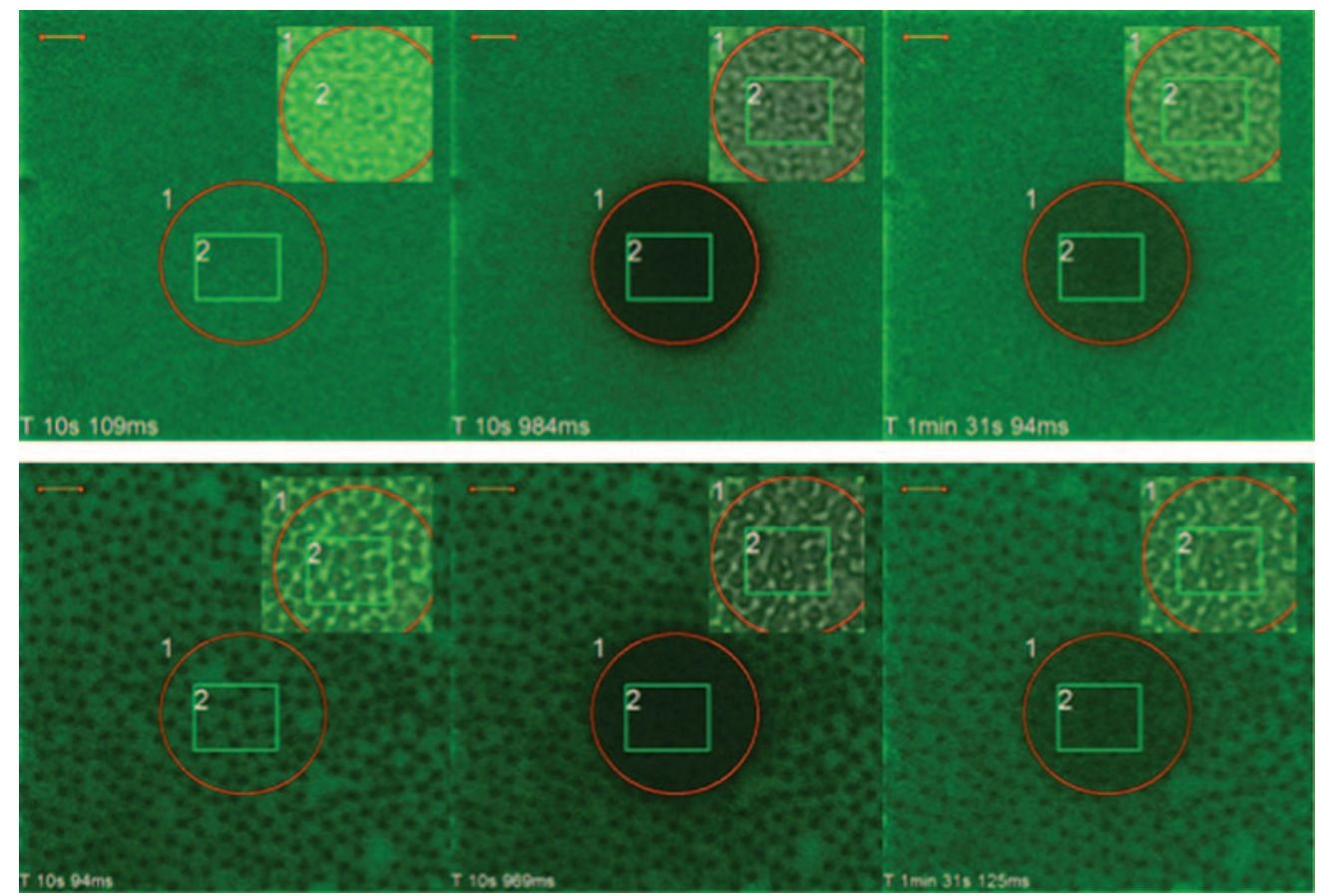

Fig. 4. Confocal microscopy images $\left(\lambda_{\mathrm{ex}}=488 \mathrm{~nm}, \lambda_{\mathrm{em}}=531 \mathrm{~nm}\right)$ of unpatterned (a) and patterned (b) $\mathrm{ZnO}$ samples after FITC-alb adsorption. Images for the montage have been picked up immediately before bleach (left), immediately after (centre) and $80 \mathrm{~s}$ after bleach (left). In the insets the merged images of fluorescence and bright field from the bleached area. Scale bar $=2 \mu \mathrm{m}$.

to colloidal lithography micropatterning and resulting in a partial $\mathrm{ZnO}$ dissolution can be invoked. ${ }^{18}$

The peak shape analysis of $\mathrm{C} 1 \mathrm{~s}$ high-resolution spectra (Fig. 3(a)) for the three investigated surfaces, namely MOCVD seed layer, $\mathrm{ZnO}$ unpatterned nanorods and $\mathrm{ZnO}$ patterned nanotubes-nanorods, evidences a similar distribution of peak components. In particular, the $\mathrm{C} 1 \mathrm{~s}$ peak is a convolution of three main different components, corresponding respectively to:

(i) $\mathrm{C}-\mathrm{C}$ and $\mathrm{C}-\mathrm{H}$ bonds (binding energy $(\mathrm{BE})=$ $284.8 \mathrm{eV}$ ),

(ii) $\mathrm{C}-\mathrm{O}$ bonds $(\mathrm{BE}=286.5 \mathrm{eV})$ and,

(iii) Carbon doubly bonded to oxygen species such as $\mathrm{C}(=\mathrm{O}) \mathrm{O}$ or $\mathrm{O}-\mathrm{C}-\mathrm{O}$ bonds $(\mathrm{BE}=288.8 \mathrm{eV})$ mainly due to surface adsorbed carbonates and hydrogen carbonates. ${ }^{19}$

On the other hand, the $\mathrm{O} 1 \mathrm{~s}$ high resolution spectra (Fig. 3(b)) clearly evidence two main peak contributions, attributed to bulk $\mathrm{Zn}-\mathrm{O}$ species $(\mathrm{BE}=530.5 \mathrm{eV})$ and to adsorbed oxygen species $(\mathrm{BE}=532.2 \mathrm{eV})$ respectively. ${ }^{20}$ The latter, clearly prevailing in the patterned sample after PS sphere removal, is an indication of a high surface hydroxylation. Therefore, it can be reasonably argued that the patterned $\mathrm{ZnO}$ nanoplatforms are characterised by a higher contribution of surface polar moieties than the corresponding unpatterned surfaces. This evidence is explained by the high surface-to-volume ratio of the present nanoplatforms where the dimensionality of the $\mathrm{ZnO}$ nanorods is combined to the porous nature of the hemispherical arrays, thus suggesting a peculiar response in terms of protein behaviour at the investigated interfaces.

FRAP experiments have been performed to get more insights about the mobility of proteins adsorbed onto the $\mathrm{ZnO}$ investigated surfaces. In this context, albumin has been used as a model biomolecule whose adsorption on solid surfaces is known to be strongly influenced by the polar character of the substrate. ${ }^{21,22}$

Some representative fluorescence images for a FRAP experiments for fluoresceine-labeled albumin adsorbed on patterned and unpatterned $\mathrm{ZnO}$ are shown in Figure 4. The fluorescence recovery, due to lateral diffusion of unbleached/bleached protein molecules inside/outside the bleached spot, is, thus, related to the protein mobility at the bio-interface between the adsorbed protein layer and the $\mathrm{ZnO}$ surface. Only a partial recovery of fluorescence is obtained from both unpatterned and patterned $\mathrm{ZnO}$ nanorods. However, the analysis of the emission intensities, normalized to the pre-bleach values (Fig. 5(a)) evidences a faster recovery for unpatterned $\mathrm{ZnO}$ nanorods than for $\mathrm{ZnO}$ nanoplatforms. Moreover, the normalised fluorescence increments for specific emission recovery related to the $\mathrm{ZnO}$ nanotubes areas (i.e., inside the micropores) is lower than that from the external $\mathrm{ZnO}$ nanorod areas (i.e., outside the micropores).

To summarise, the obtained results point to the effectiveness of chemical and/or topographical patterning of $\mathrm{ZnO}$ surfaces as a sensitive and tunable factor to control the response of biological molecules at the interface. 
(a)

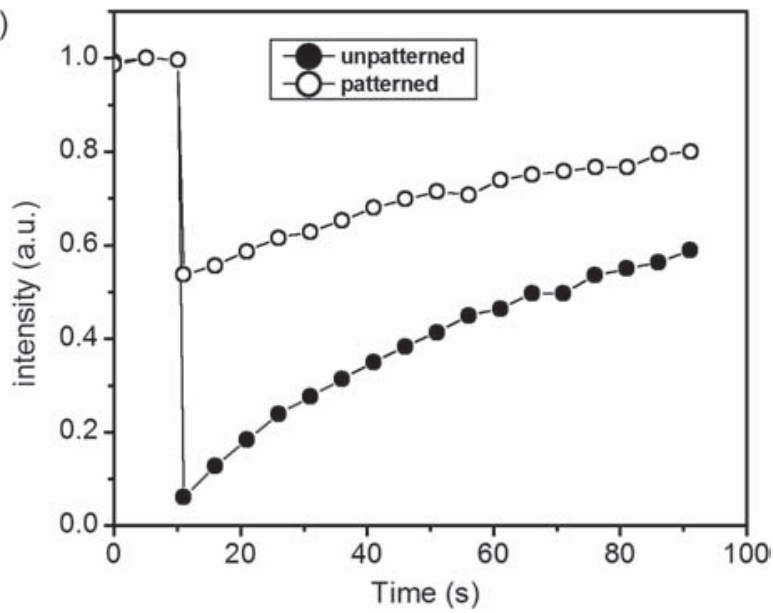

(b)

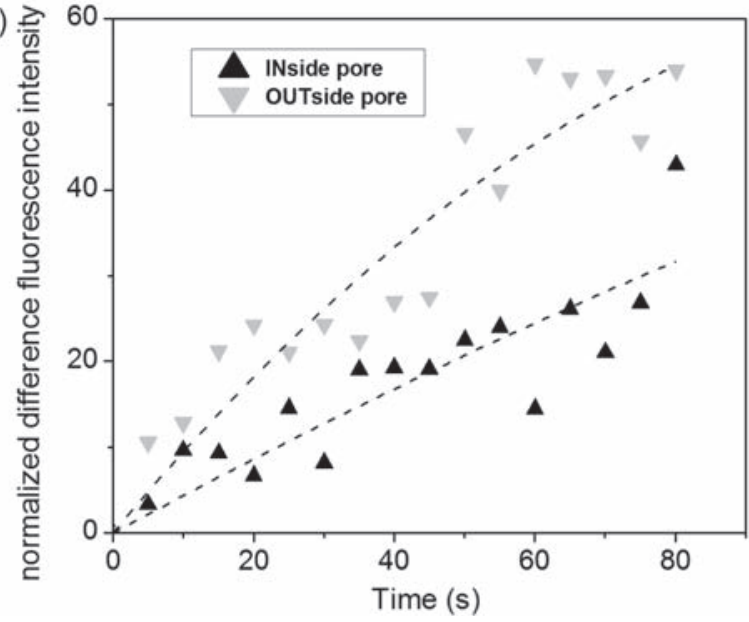

Fig. 5. (a) Normalised fluorescence intensity over whole FRAP experiments for $\mathrm{ZnO}$ unpatterned (solid circles) and patterned (open circles); (b) Normalised fluorescence increments for emission recovery from INside (up triangles) and OUTside (down triangles) $\mathrm{ZnO}$ pore areas.

\section{CONCLUSION}

Nanotube-nanorod hybrid networks have been successfully assembled as dense $\mathrm{ZnO}$ micropores arrays by an effective integration of MOCVD-CL-CBD processes. The intrinsic porosity inferred by topographical patterning affects, in turn, the surface chemical structure, as measured in terms of surface polar moieties. Proof of working measurements of protein lateral diffusion after adsorption onto the $\mathrm{ZnO}$ nanoplatforms evidence the reduced biomolecule mobility inside the hemispherical micropores, explained by their intrinsic porous nature and related polarity.

To conclude the present $\mathrm{ZnO}$ nanoplatforms have a great potential for biosensing application as their interaction with biomolecules of interest can be easily triggered by proper tuning of the system dimensionality.

Acknowledgments: Authors would like to acknowledge INSTM for support to the present study.

\section{References and Notes}

1. G. R. Patzke, Y. Zhou, R. Kontic, and F. Conrad, Angew. Chem. 50, 826 (2011)

2. W.-S. Wang, T.-T. Wu, T.-H. Chou, and Y.-Y. Chen, Nanotechnology 20, 135503 (2009).

3. A. Gurlo, Nanoscale 3, 154 (2011).

4. J.-H. Lim, C.-K. Kang, K.-K. Kim, I.-K. Park, D.-K. Hwang, and S.-J. Park, Adv. Mater. 18, 2720 (2006).

5. F. Xu, M. Dai, Y. Lu, and L. Sun, J. Phys. Chem. C 114, 2776 (2010).

6. Z. L. Wang, ACS Nano 2, 1987 (2008)

7. Dorfman, N. Kumar, and J. I. Hahm, Langmuir 22, 4890 (2006).

8. M. E. Fragalà, C. Satriano, and G. Malandrino, Chem. Comm. 7, 839 (2009).

9. M. E. Fragalà, C. Satriano, Y. Aleeva, and G. Malandrino, Thin Solid Films 518, 4484 (2010).

10. B. Weintraub, Z. Zhou, Y. Li, and Y. Deng, Nanoscale 2, 1573 (2010).

11. S.-M. Yang, S. G. Jang, D.-G. Choi, S. Kim, and H. K. Yu, Small 2, 458 (2006).

12. M. E. Fragalà and C. Satriano, J. Nanosci. Nanotechnol. 10, 5889 (2010).

13. M. E. Fragalà, G. Malandrino, M. M. Giangregorio, M. Losurdo, G. Bruno, S. Lettieri, L. Santamaria Amato, and P. Maddalena, Chem. Vap. Deposition 15, 327 (2009).

14. G. M. L. Messina, C. Satriano, and G. Marletta, Chem. Commun. 40, 5031 (2008).

15. M. E. Fragalà, Y. Aleeva, and G. Malandrino, Superlattices Microstruct. 48, 408 (2010).

16. M. E. Fragalà, Y. Aleeva, and G. Malandrino, Thin Solid Films (2011).

17. Y. B. Pyun, J. Yi, D. H. Lee, K. S. Son, G. Liu, D. K. Yi, U. Paikac, and W. I. Park, J. Mater. Chem. 20, 5136 (2010).

18. H. Q. Wang, G. H. Li, L. C. Jia, G. Z. Wang, and C. J. Tang, J. Phys. Chem. C 112, 11738 (2008).

19. K. G. Saw, K. Ibrahim, Y. T. Lim, and M. K. Chai, Thin Solid Films 515, 2879 (2007).

20. J.-C. Dupin, D. Gonbeau, P. Vinatierb, and A. Levasseurb, Phys. Chem. Chem. Phys. 2, 1319 (2000).

21. A. Auditore, C. Satriano, U. Coscia, G. Ambrosone, V. Parisi, and G. Marletta, Biomol. Eng. 19, 85 (2002).

22. G. M. L. Messina, C. Satriano, and G. Marletta, Colloids Surf. B: Biointerfaces 70, 76 (2009).

Received: 28 April 2011. Accepted: 15 July 2011. 